\title{
Calidad en la educación inicial: desafío aún pendiente en América Latina ${ }^{*}$
}

\author{
ÁNGELA MARÍA JIMÉNEZ ROJAS** \\ LUZ STELA QUINTANA HERNÁNDEZ***
}

Recibido: 25 de febrero de 2019 . Evaluado: 5 de diciembre de 2019 - Aceptado: 9 de diciembre de 2019

Citar como: Jiménez Rojas, Á. M. y Quintana Hernández, L. S. (2020). Calidad en la educación inicial: desafío aún pendiente en América Latina. Hallazgos, 17(33), 103-132. DoI: https://doi. org/10.15332/2422409X.5025

* El presente artículo es una reflexión alrededor de los resultados obtenidos por el grupo de investigación Pedainfa (Pedagogía e Infancia), en el proyecto de investigación titulado "Representaciones sociales acerca de la infancia en los futuros educadores de Licenciatura en Pedagogía Infantil de la Universidad del Quindío", financiado por Vicerrectoría de Investigaciones de la Universidad del Quindío entre 2016 y 2017.

** Doctoranda en Ciencias de la Educación. Docente de carrera en la Universidad del Quindío, Colombia.

Correo electrónico: amjimenez@uniquindio.edu.co ORCID: https://orcid.org/0000-0003-3976-7113

*** Magíster en Alta Dirección de Servicios Educativos. Docente catedrática en la Universidad del Quindío, Colombia. Docente de la Universidad de San Buenaventura, Medellín, Colombia.

Correo electrónico: 1squintana@uniquindio.edu.co

ORCID: https://orcid.org/0000-0002-0204-5291 


\section{Resumen}

El propósito de este artículo de reflexión es poner de relieve la calidad en la educación inicial con base en los atributos para la Atención y Educación de la Primera Infancia (AEPI) en América Latina. Desde los años noventa, el panorama educativo se orienta a garantizar el derecho a la educación igualitaria y de calidad, focalizando los esfuerzos en la infancia. El artículo tiene cuatro apartados. El primero caracteriza la situación del sistema educativo latinoamericano en los años ochenta y noventa, que gesta los marcos de referencia para la calidad en la educación inicial en la actualidad. El segundo describe los conceptos de calidad educativa y educación inicial, y resalta el derecho a la educación. El tercero se centra en la reflexión sobre diversas connotaciones del concepto de infancia y primera infancia. El cuarto argumenta la educación inicial desde los atributos y principales desafíos para la atención y educación de calidad. Finalmente, se exponen las reflexiones que convocan a continuar trabajando como docentes e investigadores alrededor de dicha temática.

Palabras clave: América Latina, calidad de la educación, educación de la primera infancia, infancia, primera infancia. 


\section{Quality in preschool education: challenge still pending in Latin America}

\section{Abstract}

The purpose of this reflection article is to highlight the quality of preschool education based on the attributes for Early Childhood Care and Education (ECCE) in Latin America. Since the 1990s, the educational overview is aimed at guaranteeing the right to equal and quality education, focusing efforts on childhood. The article has four sections. The first characterizes the situation of the Latin American education system in the 1980s and 1990s, which develops the frames of reference for quality in preschool education today. The second describes the concepts of educational quality and preschool education, and highlights the right to education. The third focuses on the reflection on various connotations of the concept of childhood and early childhood. The fourth discusses preschool education from the attributes and main challenges for quality care and education. Finally, the reflections that call to continue working as teachers and researchers around this subject are exposed.

Keywords: Latin America, quality of education, early childhood education, childhood, early childhood.

\section{Qualidade na educação inicial: desafio ainda pendente na América Latina}

\section{Resumo}

O propósito deste artigo de reflexão é destacar a qualidade na educação inicial baseado nos atributos para a Atenção e Educação da Primeira Infância (AEPI) na América Latina. Desde os anos noventa, o panorama educativo orienta-se a garantir o direito à educação igualitária e de qualidade, focando os esforços na infância. $\mathrm{O}$ artigo tem quatro partes. A primeira caracteriza a situação do sistema educativo latino-americano nos anos oitenta e noventa, que gera os marcos de referência para a qualidade na educação inicial na atualidade. A segunda descreve os conceitos de qualidade educativa e educação inicial, e resalta o direito à educação. A terceira foca-se na reflexão sobre diversas conotações do conceito de infância e primeira infância. A quarta argumenta a educação inicial desde os atributos e principais desafios para a atenção e educação de qualidade. Finalmente, se expõem as reflexões que convocam a continuar trabalhando como docentes e pesquisadores acerca dessa temática.

Palavras-chave: América Latina, qualidade da educação, educação da primeira infância, infância, primeira infância. 


\section{Introducción}

La calidad en la educación inicial actualmente es insignia en las políticas y reformas educativas en las regiones latinoamericanas. En estas se reconoce que la educación es un derecho universal desde el nacimiento, que debe garantizar el desarrollo infantil, la igualdad y la equidad. Hace ya dos décadas que el concepto se promueve en escenarios internacionales por medio de organismos multilaterales, cuyo impacto y transformación aún se están gestando. Estos organismos han suscitado reflexiones sobre la relación que hay entre la educación inicial y los planteamientos de las políticas educativas en América Latina, y han reafirmado su interrelación para alcanzar la meta educativa: educación para todos.

En este artículo se analiza dicha interrelación, develando así la complejidad de los conceptos de calidad educativa, educación inicial, infancia y primera infancia, que requieren en la región latinoamericana mayor reflexión y compromiso para el cumplimiento de su meta educativa. En diversos eventos internacionales se han trazado directrices, marcos de referencia y propuestas de acción que señalan la importancia de reflexionar e identificar cómo se concibe, se desarrolla y se realiza el seguimiento a la calidad en la educación inicial. En tales convenciones se resalta la idea de que la atención y la educación de la primera infancia (AEPI) convocan a una coconstrucción y responsabilidad compartida, entre otros, con la comunidad educativa y los contextos social, cultural, político y económico.

Con esta indagación también se pretende revelar la convergencia de la calidad en la educación inicial desde la perspectiva enmarcada por la Conferencia Mundial sobre la Atención y Educación de la Primera Infancia, celebrada en Moscú (2010). Allí se enunciaron los atributos acerca del cuidado, la atención y el desarrollo infantil. Se develó que la educación inicial debe construir y potencializar escenarios que fortalezcan dichos atributos, teniendo como precedente la complejidad de la primera infancia, enmarcada en contextos histórico-culturales dinámicos que le otorgan particularidades y connotaciones diversas a la región latinoamericana.

\section{Sistema educativo latinoamericano a comienzos de los años noventa}

Las particularidades y crisis afrontadas en el sistema educativo de América Latina en las décadas de los setenta y ochenta, marcadas por un excesivo centralismo, falta de 
equidad y calidad en el servicio educativo, insuficiente financiamiento, poca autonomía y escasa participación de la comunidad educativa en la gestión del proceso educativo, entre otros, llevaron a crear un escenario propicio para el diseño de políticas y reformas educativas basadas en amplios consensos y compromisos nacionales e internacionales. Tales políticas tenían como carta de navegación renovar los sistemas educativos, otorgándoles la atención y el lugar prioritario en la agenda política de los países latinoamericanos, lo que dio respuesta a las necesidades regionales y a la economía global. Estos cambios se vieron permeados por la transformación del concepto desarrollo humano, que hasta los años ochenta estuvo centrado en la dimensión económica basada en el aumento de la renta per cápita y de la producción, indicadores que medían el desarrollo en términos cuantitativos. En este sentido, Bedoya (2010) afirma que "el enfoque de desarrollo suponía un vínculo estrecho entre el crecimiento económico nacional y la ampliación de las opciones individuales del ser humano” (p. 280). Sin embargo, la realidad mostró que los indicadores de satisfacción de necesidades básicas no mejoraban y, por ende, no se había avanzado en la consecución del desarrollo.

En la década de los noventa, el Programa de las Naciones Unidas para el Desarrollo (PNUD), basado en los aportes de Amartya Sen, definió el desarrollo como el proceso que amplía las opciones de las personas y mejora las capacidades humanas, es decir, la diversidad de cosas que las personas pueden hacer o ser en la vida, y las libertades para vivir una vida digna, larga y saludable, tener acceso a la educación y participar en la vida de su comunidad y en las decisiones que las afecten (citado por Bedoya, 2010, p. 280). Como resultado de esto, el PNUD (2000) sustenta que los fines del desarrollo se orientan al "fortalecimiento de determinadas capacidades relacionadas con toda la gama de cosas que una persona puede ser y hacer en su vida" (Cejudo, 2006, p. 366).

Los anteriores aspectos llevan a resaltar el papel de la educación en el desarrollo de capacidades del ser humano, que según el PNUD (2010) “es el proceso mediante el cual las personas, organizaciones y sociedades obtienen, fortalecen y mantienen las aptitudes necesarias para establecer y alcanzar sus propios objetivos de desarrollo a lo largo del tiempo” (p. 3). En este aspecto, las políticas educativas de la década de los noventa, según Gajardo (1999), hicieron hincapié en dos grandes elementos orientadores: la mejora de la calidad de la educación y una mayor equidad en la distribución de las oportunidades educativas.

En este sentido, la oleada de políticas y estrategias educativas compartidas por Latinoamérica evidencia las particularidades de orden cultural, social, económico y político que enmarcan a cada una de las regiones y contribuyen a cristalizar las 
reformas educativas. Algunos de los elementos centrales de estos hechos son: el fortalecimiento de la calidad educativa, el acceso, la equidad, la distribución equitativa de oportunidades a favor de los niños, las niñas y los más pobres y vulnerables, las innovaciones y transformaciones pedagógicas, la descentralización y el fortalecimiento de la gestión educativa y de las condiciones de trabajo docente. En esta misma línea, Corrales (1999) señala:

La prioridad en la educación tiene dos objetivos principales, por una parte, el mejoramiento de la calidad de la educación que se ve como una fuente de competitividad económica de los países [...] Un segundo objetivo, se refiere a que la educación de alta calidad ha pasado a ser un sinónimo de desarrollo nacional autosostenido, no solo de competitividad internacional. (p. 3)

Con base en este referente instalado y desarrollado con gran fuerza en los años noventa, la educación, en especial la inicial, adquiere una connotación prioritaria e innegociable para el desarrollo de los países, lo cual la convierte en el foco de las reformas internacionales y, por supuesto, latinoamericanas. Esta connotación continúa en el nuevo siglo.

En septiembre de 2000, en la Cumbre del Milenio realizada por los ciento ochenta y nueve países miembros de la Organización de las Naciones Unidas (ONU), se definió la Declaración del Milenio (ONU, 2000), documento que además de confirmar el deseo de lograr un "mundo más pacífico, más próspero y más justo” (p. 1), reconoce su responsabilidad con las sociedades. Al establecer dicha declaración, los Estados miembros fijaron los Objetivos de Desarrollo del Milenio (ODM). Tales objetivos tuvieron como fecha límite diciembre de 2015, y proporcionaron un marco para que los organismos que forman parte de la onU trabajen conjuntamente hacia un fin común. De esta manera se comprometieron a tomar nuevas medidas y aunar esfuerzos en "combatir la pobreza, el hambre, las enfermedades, el analfabetismo, la degradación del ambiente y la discriminación contra la mujer" (Centro de Información de las Naciones Unidas, s. f., párr. 1 y 2).

En este punto es de señalar que en noviembre de 2015 se realizó la cumbre para definir los objetivos que guían las acciones de los países miembros de la onU desde enero de 2016 hasta 2030. Estos se conocen como Objetivos de Desarrollo Sostenible (ODS), que se constituyen en una agenda que realiza "un llamado universal para adoptar medidas orientadas a colocar fin a la pobreza, proteger el planeta y garantizar que todas las personas gocen de paz y prosperidad” (PNUD, s. f., párr. 1). La agenda plantea diecisiete objetivos en los que es evidente la mirada hacia la infancia, ya que se busca 
"satisfacer las necesidades del presente sin comprometer las necesidades de las futuras generaciones" (ONU y Bruntland Commission, citados por Chavarro et ál., 2017), es decir, se busca mejorar la calidad de vida, el cuidado ambiental y nuevas opciones de desarrollo económico. Al centrar la mirada en la educación, se encuentra el cuarto objetivo: "Garantizar una educación inclusiva y equitativa de calidad y promover oportunidades de aprendizaje permanente para todos”, cuya segunda meta pretende,

[...] de aquí a 2030, asegurar que todas las niñas y todos los niños tengan acceso a servicios de atención y desarrollo en la primera infancia y educación preescolar de calidad, a fin de que estén preparados para la enseñanza primaria. (onU y Cepal, 2018, p. 28)

De esta meta se espera que en la primera infancia se adquieran las competencias en todas las dimensiones del desarrollo, que posibiliten la adaptación de los niños y las niñas a los cambios y desafíos que tendrán en su vida.

\section{La calidad educativa en la educación inicial}

Para describir la calidad educativa en la educación inicial, es relevante precisar a qué alude el concepto de calidad educativa. En este sentido, es necesario indagar inicialmente sobre qué es calidad, la cual se define como una "propiedad o conjunto de propiedades inherentes a algo, que permiten juzgar su valor” (Real Academia Española, 2019). Dicha definición suscita otro interrogante: cuando se enuncia el concepto de calidad educativa en la educación inicial, ¿a qué grupo de propiedades se hace referencia en el momento de su valoración? Este es, precisamente, el punto de partida para aclarar que solo después de la década de los ochenta este concepto encuentra utilidad y aplicación en el sistema educativo; fue extrapolado de las ciencias económicas y administrativas para denotar productividad, producto final y eficientísimo. Al respecto, Aguerrondo (2009) afirma:

La aparición del concepto calidad de la educación se produjo históricamente dentro de un contexto específico. Viene de un modelo de calidad de resultados, de calidad de producto final, que nos pone en guardia, sobre todo, del hecho de que bajo estas ideas suelen estar los conceptos de la ideología de eficiencia social que considera al docente poco menos que como un obrero de línea que emplea paquetes instruccionales, cuyos objetivos, actividades y materiales le llegan prefabricados, y en el cual la calidad se mide por fenómenos casi aislados, que se recogen en el producto final. (p. 2) 
El origen del concepto de calidad educativa es reciente en la literatura pedagógica, y más aún en la educación inicial, escenario en el cual se denota con mayor rigor su carácter amplio, complejo y polisémico. El concepto involucra políticas; reformas y programas educativos; autoridades y agentes educativos; procesos de planificación, organización y gestión del currículo; proyectos educativos y pedagógicos, además de las estrategias didácticas. Tiene en cuenta la participación activa de los niños, los maestros, la familia y la comunidad educativa, agentes que reflejan las realidades de los contextos socioculturales. Por tal razón, no existe una única definición ni visión acerca de la calidad educativa, lo que complejiza la naturaleza del concepto y confirma la influencia del entorno sociocultural.

Así, existen diversas definiciones acerca de la calidad educativa, algunas de las cuales se sintetizan en la tabla 1.

Tabla 1. Definiciones de calidad educativa

\section{Definición calidad educativa}

"La escuela de calidad es aquella que promueve el progreso de los estudiantes en una amplia gama de logros intelectuales, sociales, morales y emocionales, teniendo en cuenta su nivel socioeconómico, su medio familiar y su aprendizaje previo. Un sistema escolar eficiente es el que maximiza la capacidad de las escuelas para alcanzar estos resultados. Lo que supone adoptar la noción de valor añadido de la eficacia escolar" (Mortimore, citado por Marchesi, 2014, p. 43).

"La calidad educativa es un concepto complejo, que incluye tanto la riqueza y adecuación de los insumos, la relevancia y pertinencia curricular, como los procesos de enseñanza-aprendizaje y los resultados de estos procesos" (Cariola, 2013, p. 61).

"Una educación capaz de producir aprendizajes útiles para la vida actual y futura de sus educandos en las esferas personal, familiar, cívica y económico-laboral” (Schmelkes, 2009, p. 50).

“Es la congruencia o consistencia entre los ejes fundamentales (ideológicos, políticos, pedagógicos, etc.) y la organización (o la apariencia fenoménica) del aparato educativo, no se percibe inconsistencia” (Aguerrondo, 2009, p. 3).

“Es una construcción social, históricamente determinada, cuya concepción obedece a condiciones económicas, políticas, culturales y sociales determinadas” (Guzmán, 2005, p. 3).

"El concepto de 'calidad educativa' es complejo y entraña diversas dimensiones que lo articulan en una unidad verdaderamente integral” (Seibold, 2000, p. 217).

Fuente: elaboración propia 
La tabla 1 evidencia las concepciones que se tienen de la calidad educativa; muestra su indefinición y variedad y la importancia del contexto educativo en el cual se valida y sustenta. En este sentido, la Organización para la Cooperación y el Desarrollo Económicos, en 1991, en el Informe internacional de las escuelas y calidad de la enseñanza, sostiene que la "calidad significa cosas diferentes para distintos observadores y grupos de interés; no todos comparten las mismas percepciones de las prioridades para un cambio" (citado por Tiana, 2009, p. 116). En esta misma línea, Bodero (2014) señala que las dificultades para plantear una única definición del término calidad educativa puede deberse a aspectos como:

- La educación en sí misma se convierte en una realidad compleja al centrarse en el abordaje del ser humano en todas sus dimensiones del desarrollo.

- Al plantear las definiciones de educación, se deben reconocer las diferentes conceptualizaciones, miradas y modelos que llevan a plantear diversas metas.

- Las acciones del educador están mediadas por sus convicciones y saber pedagógico.

Es evidente que las definiciones de calidad en educación son múltiples. Por esta razón, la Unesco señala que la educación es un asunto político. Así, al hablar de calidad, se deben tener en cuenta los derechos humanos y las obligaciones del Estado. Por eso no puede entenderse la calidad educativa como un proceso productivo o mediante un sistema de proveedor-agente, sino que debe analizarse sobre la base de cinco aspectos esenciales: relevancia, pertinencia, equidad, eficacia y eficiencia; la falta de alguno de estos establece una idea sesgada del concepto (Murillo y Cuenca, 2007).

Según Rodríguez (citado por el Ministerio de Educación Nacional [MEN], 2010), las diferencias en la concepción de la calidad educativa cobran importancia al revisar cuatro componentes en los que se busca la calidad: gestión pedagógica, cobertura, resultados y gestión educativa. Por su parte, Toranzos (citada por el MEN, 2010) expuso que se deben abordar tres dimensiones de calidad: eficacia, es decir, la educación en la que los estudiantes aprenden lo que se supone deben aprender; relevancia social e individual de lo asimilado en el sistema educativo, y calidad de los procesos y medios empleados para que los estudiantes desarrollen su experiencia educativa (MEN, 2010, p. 8).

De otro lado, Delors (1996) planteó que al hablar de educación de calidad se debe recalcar que es un proceso de aprendizaje que se da a lo largo de la vida y que debe ser pertinente. Señala los siguientes como los aprendizajes fundamentales para cada persona: "aprender a conocer, [...] aprender a hacer, [...] aprender a ser [...] y aprender a vivir juntos” (p. 91). Así, se hace hincapié en que la calidad puede influir en lo 
personal y en lo que respecta al desarrollo de una sociedad. De este modo, la calidad es vista como la necesidad de respetar los derechos como es debido, razón por la cual se debe dar compromiso social, cultural, político y económico de la sociedad-Estado, en pro de la calidad educativa y el abordaje de las exigencias sociales.

En relación con lo anterior, para mirar la calidad en el ámbito educativo es necesario recordar que la educación, a través de la historia, es un aspecto fundamental en el desarrollo de las sociedades. En este sentido, como plantea Reimers (2000), "las sociedades sostienen las escuelas por razones que se encuentran entre dos extremos: por una parte, porque contribuyen a reproducir el orden social; por otra, por la esperanza de que pueden contribuir a mejorar este orden” (p. 12). Esto se evidencia con base en las políticas planteadas a escala mundial, dirigidas a promover la igualdad de oportunidades en el ámbito educativo, y de las cuales cobran gran relevancia las dirigidas a la población infantil, lo que amerita aclarar el término educación inicial.

El concepto de educación inicial ha trasegado por varios senderos. En esta vía, ha adoptado nombres como educación preprimaria, educación parvularia, educación preescolar, educación infantil y educación en la primera infancia. La trascendencia del concepto se da desde 1990 con la Conferencia Mundial de Educación para Todos, en Jomtien (Tailandia). Posteriormente se reafirma en marzo de 2000, en Chile, con la Declaración del Simposio Mundial de Educación Parvularia o Inicial, enmarcada como educación inicial para el siglo xxi, y en abril de 2000, en Dakar (Senegal), con el Foro Mundial sobre la Educación. Aquí se recuerda que la educación inicial empieza mucho antes que el sistema educativo formal. También se ratifica que desde el momento del nacimiento, los niños y las niñas adquieren el derecho a la educación y con este al pleno desarrollo de sus potencialidades; se resalta que el periodo prenatal es decisivo para los posteriores desarrollos en el ser humano. Por tal razón, los procesos de aprendizaje inician desde el nacimiento y, en consecuencia, la educación inicial posee un efecto positivo en la disminución de las desigualdades económicas, sociales y culturales. Es de señalar que la "educación inicial se refiere al proceso de enseñanza-aprendizaje durante la primera infancia haciendo énfasis en contenidos, el desarrollo de conductas, destrezas, competencias y la integración del infante en su entorno" (Marco, 2014, p. 19).

Teniendo en cuenta que desde los noventa se destaca la educación como la única y oportuna respuesta a las demandas de Latinoamérica y del contexto internacional, la educación inicial o de la primera infancia se revela como un derecho 
de todas las personas, no como un privilegio o exclusividad de una región, de unos pocos niños o de unas comunidades. Esto se evidencia en la Declaración Universal de los Derechos Humanos (onu, 1948), en cuyo artículo 2 se afirma que todos los seres humanos son iguales en derechos, y en el artículo 26, parágrafo 2, se establece que el objeto de la educación es el pleno desarrollo de la personalidad y el fortalecimiento del respeto a los derechos humanos. En la Declaración se promulga:

Toda persona tiene derecho a la educación. La educación debe ser gratuita, al menos en lo concerniente a la instrucción elemental y fundamental. La instrucción elemental será obligatoria. La instrucción técnica y profesional habrá de ser generalizada, el acceso a los estudios superiores será igual para todos, en función de los méritos respectivos. (ONU, 1948, art. 26)

Así mismo, el derecho a la educación es inalienable, innegociable e intransferible, y debe hacerse valer desde la niñez. Al respecto, "los Estados parte reconocen el derecho del niño a la educación” (Unicef, 2006, p. 22). La educación como garante del desarrollo integral desde la infancia, en especial la primera infancia, debe formar sin ningún tipo de discriminación ni exclusión. Se tiene que enmarcar desde un contexto sociocultural para favorecer y fomentar una sociedad libre, tolerante, solidaria, participativa y democrática. Al ejercer las condiciones de igualdad de oportunidades educacionales, se concibe la educación inicial desde el marco jurídico y social del derecho a la educación, es decir, no se limita exclusivamente a otorgar el acceso y la permanencia al sistema escolar. En este sentido, como plantea Blanco (2014), el derecho a la educación debe promover el máximo desarrollo de cada persona, al igual que el aprendizaje, y que cada niño o niña acceda a las escuelas de la comunidad cercana en condiciones iguales a los demás niños.

En Colombia, desde la Política Pública para la Primera Infancia (Consejo Nacional de Política Económica Social 109, 2007) se entiende la educación inicial como

[...] un proceso continuo y permanente de interacciones y relaciones sociales de calidad, oportunas y pertinentes que posibilitan a los niños y las niñas potenciar sus capacidades y adquirir competencias para la vida, en función de un desarrollo pleno que propicie su constitución como sujetos de derechos. Esto implica realizar un cuidado y acompañamiento afectuoso e inteligente del crecimiento y desarrollo de los niños y las niñas, en ambientes de socialización sanos y seguros para que logren aprendizajes de calidad. (p. 23) 
Al realizar un análisis de lo expuesto en este apartado acerca de la calidad educativa y las concepciones de educación inicial, se hallan contradicciones. En la revisión de las nociones de la calidad educativa, articulada a los oDs, existe una fragmentación de la mirada del ser humano, puesto que hay un objetivo centrado en educación, cuando debería encontrarse la articulación entre los diecisiete objetivos. Además, se concibe la educación como el ámbito desde el cual se da un orden social, y se espera que cumpla con los lineamientos o estándares dados por el Estado para cada uno de los niveles y áreas que se abordan. Sin embargo, se desconoce que el gran objetivo de la educación se debe centrar en acompañar y facilitar los procesos que permitan a los niños y las niñas llegar a ser lo mejor que puedan ser.

En otras palabras, el sistema educativo continúa centrado en la transmisión de conocimientos que se vean reflejados en las pruebas de Estado y en las evaluaciones internacionales (como las PISA). Tales pruebas realizan una especie de tamizaje que clasifica a los estudiantes en buenos, regulares o malos, según sus resultados. Se olvida, entonces, que lo esencial para los estudiantes es adquirir los valores que les permitan identificar lo positivo y lo negativo, y con ello la autonomía, de tal forma que se construyan buenas relaciones. Estas pruebas no están enfocadas en que los estudiantes puedan consolidar un pensamiento crítico para analizar las situaciones que enfrentan, y así cimentar los sueños que orientan su desarrollo, es decir, contribuir al ser para que sea de calidad.

Parece que existe una discontinuidad en el proceso de desarrollo del ser humano, ya que las orientaciones para abordar la educación en la primera infancia están centradas en los niños. Estas pretenden un ambiente seguro que permita el reconocimiento de cada uno y en el que desarrollen aprendizajes significativos con base en las actividades propias de la infancia (juego, arte, literatura, exploración del medio). No obstante, desde la primaria, la calidad se articula con la cobertura y la reproducción de conocimiento, lo que la reduce a las definiciones del sector productivo, centrados en la competencia y la igualdad, dejando de lado la diferencia de cada ser humano.

\section{La infancia y la primera infancia}

Antes de continuar argumentando la relevancia de la calidad educativa en la educación para la primera infancia o la educación inicial, es necesario definir el concepto 
de primera infancia y diferenciarlo del de infancia. "La noción de infancia tiene un carácter histórico y cultural y es por ello que ha tenido apreciaciones en la historia, su concepción depende del contexto cultural de la época” (Jaramillo, 2007, p. 110). Las concepciones de infancia están enmarcadas según las particularidades políticas, económicas, educativas, socioculturales de las regiones latinoamericanas. En la tabla 2 se presenta el recorrido del concepto a través de la historia, tomando como referente los planteamientos de Santos (citado por Jaramillo, 2007, p. 111).

Tabla 2. Línea del tiempo del concepto y abordaje de la infancia

\begin{tabular}{|c|c|}
\hline \multicolumn{2}{|r|}{ Cronología en el abordaje de la infancia } \\
\hline Época & Característica \\
\hline $\begin{array}{l}\text { Años } 354-430 \\
\text { hasta el siglo IV }\end{array}$ & $\begin{array}{l}\text { Los niños se caracterizan por ser indefensos y, por tal razón, requieren la pre- } \\
\text { sencia de un adulto para su cuidado y protección. }\end{array}$ \\
\hline Siglo xv & Los niños son una propiedad, debido a la atención que necesitan. \\
\hline Siglo XVI & $\begin{array}{l}\text { Empieza una mirada hacia el niño como ser humano pero carente de habilida- } \\
\text { des. El niño, por tal razón, es asumido como un adulto pequeño. }\end{array}$ \\
\hline Siglos XVI y XVII & $\begin{array}{l}\text { Surge el reconocimiento del niño desde una perspectiva de bondad e inocen- } \\
\text { cia y como poseedor de condiciones innatas. }\end{array}$ \\
\hline Siglo XVIII & $\begin{array}{l}\text { Se categoriza y clasifica como infante, discriminando el término en un faltante } \\
\text { para ser alguien dentro de la sociedad. }\end{array}$ \\
\hline Siglo $\mathrm{xx}$ & $\begin{array}{l}\text { Evidencia diversos esfuerzos por transformar y respetar el espacio que mere- } \\
\text { cen. Se reconoce el niño como un sujeto social de derecho. }\end{array}$ \\
\hline Siglo XXI & $\begin{array}{l}\text { La Unesco y la Convención Internacional de los Derecho del Niño han estado } \\
\text { presentes y activas de acuerdo con los movimientos modernos y contemporá- } \\
\text { neos, para redefinir al niño como sujeto de derecho y otorgarle un lugar espe- } \\
\text { cial dentro de la organización social, como persona y como ciudadano. }\end{array}$ \\
\hline
\end{tabular}

Fuente: elaboración propia

En el recorrido expuesto en la tabla 2, se evidencia que la evolución y el abordaje del concepto de infancia responde a los tiempos y momentos históricos sociales tanto en Latinoamérica como en el mundo. Por lo tanto, existen múltiples definiciones; en la tabla 3 se presentan algunas. 
Tabla 3. ¿Qué es la infancia?

\begin{tabular}{|c|}
\hline ¿Qué es la infancia? \\
\hline $\begin{array}{l}\text { “Es una categoría social [...] porque cada sociedad, cada cultura define explícita o implícitamente } \\
\text { que es infancia, cuáles son sus características, y, en consecuencia, qué períodos de la vida incluye. } \\
\text { [...] sin que ello signifique que es menos importante, la infancia no es sólo un período de vida, sino } \\
\text { que se refiere también a un conjunto de población de un territorio, que reúne las características de } \\
\text { estar en tal periodo” (Casas, citado por Alzate, 2003, p. 117). }\end{array}$ \\
\hline $\begin{array}{l}\text { "Las concepciones, imágenes o figuras que tenemos de la infancia tienen una estrecha vinculación } \\
\text { con los cambios históricos y con los modos de organización socioeconómicos y cultural de las so- } \\
\text { ciedades (Ariés, Becchi, Julia, Gélis, Muñoz y Pachón), con las formas o pautas de crianza (De Mau- } \\
\text { se), con los intereses sociopolíticos (Varela), con el desarrollo de las teorías pedagógicas (Escolano, } \\
\text { Narodowski, Saldarriaga); así como con el reconocimiento de los derechos de la infancia en las } \\
\text { sociedades occidentales y con el desarrollo de políticas sociales al respecto (García y Carranza), } \\
\text { con las representaciones sociales que las sociedades y los sujetos adultos tenemos de ella, ya sea } \\
\text { como una realidad social no necesariamente objetiva ni universal, sino ante todo, como consenso } \\
\text { social aceptado (Casas, Chombart de Lauwe)” (citados por Álzate, 2003, p. 13). }\end{array}$ \\
\hline $\begin{array}{l}\text { "Es algo más que el tiempo que transcurre entre el nacimiento y la llegada de la edad adulta, se } \\
\text { refiere al estado y la condición de la vida de un niño, a la calidad de esos años” (Bellamy, 2004, p. 1). }\end{array}$ \\
\hline $\begin{array}{l}\text { "Es la etapa en la que los niños y niñas tienen que estar en la escuela y en los lugares de recreo, } \\
\text { crecer fuertes y seguros de sí mismos y recibir el amor y el estímulo de sus familias y de una comu- } \\
\text { nidad amplia de adultos” (Unicef, 2005, párr. 1). }\end{array}$ \\
\hline $\begin{array}{l}\text { “Es una realidad construida, que se configura y reconfigura a partir de elementos culturales, histó- } \\
\text { ricos, políticos y sociales” (García y Gallego, 2011, p. 18). }\end{array}$ \\
\hline $\begin{array}{l}\text { “Es una construcción sociocultural diversa y cambiante a lo largo del tiempo (p. 8) [...] Se construye } \\
\text { desde distintas cosmovisiones, por lo que es necesario comprenderla considerando la intercultu- } \\
\text { ralidad” (Muñoz, 2012, p. 12). }\end{array}$ \\
\hline
\end{tabular}

Fuente: elaboración propia

De esta manera, al asumir la infancia como una categoría configurada y recreada en diversos momentos históricos, surgen algunos interrogantes como: ¿cuál es la diferencia entre el concepto de infancia y la primera infancia o infancia temprana?, ¿cuál es el marco internacional que legitima tanto la infancia como la primera infancia? En la tabla 4 se exponen algunas de las definiciones del concepto de primera infancia o infancia temprana. En la tabla 5 se aprecia una síntesis del marco internacional en el reconocimiento de la infancia y primera infancia. 


\section{Definición primera infancia}

La infancia se refiere a una persona menor de 18 años que necesita cuidados especiales desde antes del nacimiento (Unicef, 1989, art. 1).

"Periodo que inicia desde el nacimiento, en el cual se cimientan las bases biológicas, neurológicas, cognitivas, socio-afectivas que propiciarán el desarrollo integral del individuo, es pilar de aprendizajes posteriores” (Unesco, 1999).

"Integrada por la población menor de 6 años, aunque algunos países incluyen en este grupo a los menores de 8 años de edad [...] etapa inicial del ciclo vital, durante la que estructuran las bases para el desarrollo cognitivo, emocional y social del ser humano” (Romero, 2009, p. 40).

"Etapa del ciclo vital en la que se establecen las bases para el desarrollo cognitivo, emocional y social del ser humano. Comprende la franja poblacional que va de los cero a los seis años" (Colombia, 8 de noviembre de 2006, art. 29).

"Desde el nacimiento, el primer año de vida, el periodo pre-escolar hasta la transición hacia el periodo escolar” (Comité de los Derechos del Niño, citado por Unesco y OEI, 2010, p. 13).

"Es una edad delicada que se caracteriza por la rapidez de la evolución física, cognitiva, lingüística, social y afectiva” (Unesco, 2010a, p. 2).

"Es un periodo altamente sensible, durante el cual se sientan las bases para un desarrollo adecuado y la capacidad de aprendizaje a lo largo de la vida” (Unesco, 2014, p. 49).

"Primera infancia va desde la gestación, pasando por el nacimiento, hasta los 8 años de edad [...] es una etapa crucial para el desarrollo de los niños y niñas [...] en la que experimentan rápidos y profundos cambios, pues pasan de estar dotados de las capacidades elementales para la sobrevivencia y de una amplia gama de potencialidades, a dominar complejas habilidades físicas, emocionales, psíquicas, cognitivas y sociales [...]. Por esto, la primera infancia, y en particular el periodo que abarca las edades de 0 a 3 años, es cualitativamente más que el comienzo de la vida; es, en realidad, el cimiento de esta” (Unicef, s. f., párr. 1, 3, 5).

Fuente: elaboración propia 
Tabla 5. Marco internacional en el reconocimiento de la infancia y de la primera infancia

\begin{tabular}{|c|c|}
\hline Año & Evento \\
\hline 1919 & Reconocimiento jurídico internacional de los Derechos de la Niñez. \\
\hline 1924 & Declaración de Ginebra sobre los Derechos del Niño aprobada por la Sociedad de Naciones. \\
\hline 1948 & $\begin{array}{l}\text { Declaración de los Derechos Humanos, aprobada por la Asamblea General de las Nacio- } \\
\text { nes Unidas. }\end{array}$ \\
\hline 1959 & $\begin{array}{l}\text { Declaración sobre los Derechos del Niño aprobada por la Asamblea General de Naciones } \\
\text { Unidas. }\end{array}$ \\
\hline 1979 & $\begin{array}{l}\text { La Organización de las Naciones Unidas declara este año como el Año Internacional del } \\
\text { Niño. }\end{array}$ \\
\hline 1989 & $\begin{array}{l}\text { La Asamblea General de las Naciones Unidas aprueba la Convención sobre los Derechos } \\
\text { del Niño. }\end{array}$ \\
\hline 1990 & Se celebra la Cumbre Mundial en favor de la Infancia. \\
\hline 1990 & Declaración Mundial sobre la Educación para Todos. \\
\hline 1994 & Se declara el Año Internacional de la Familia. \\
\hline 1999 & $\begin{array}{l}\text { Se aprueba el Convenio sobre la Prohibición de las Peores Formas de Trabajo Infantil y la } \\
\text { Acción Inmediata para su Eliminación. }\end{array}$ \\
\hline 2000 & Foro Mundial sobre la Educación, en Dakar. \\
\hline 2000 & $\begin{array}{l}\text { Cumbre del Milenio de la Organización de las Naciones Unidas, Objetivos del Desarrollo } \\
\text { del Milenio. }\end{array}$ \\
\hline 2000 & Simposio Mundial de Educación Parvularia o Inicial. Educación inicial para el siglo xxi. \\
\hline 2002 & Sesión especial de la Asamblea General de las Naciones Unidas en favor de la infancia. \\
\hline 2007 & Compromiso Hemisférico por la Educación de la Primera Infancia. \\
\hline 2007 & $\begin{array}{l}\text { Creación del Instituto de Desarrollo e Innovación Educativa de Primera Infancia y Dere- } \\
\text { cho de la Niñez. }\end{array}$ \\
\hline 2008 & Conferencia Iberoamericana de Ministerios de Educación. \\
\hline 2009 & Foro Mundial Grupos de Trabajo por la Primera Infancia Sociedad Civil-Estado. \\
\hline 2009 & Establecimiento de las Metas Educativas 2021. \\
\hline 2010 & $\begin{array}{l}\text { Cumbre Iberoamericana, compromiso con la atención integral a la Pedagogía Infantil y } \\
\text { garantizar su calidad. }\end{array}$ \\
\hline 2010 & $\begin{array}{l}\text { Conferencia Mundial sobre Atención y Educación de la Primera Infancia (AEPI): Construir } \\
\text { la riqueza de las naciones. }\end{array}$ \\
\hline 2011 & Plan regional de Atención integral a la Primera Infancia (Praipi). \\
\hline
\end{tabular}




\begin{tabular}{|c|l|}
\hline Año & \multicolumn{1}{|c|}{ Evento } \\
\hline 2012 & Encuentro Internacional de Educación Inicial y Preescolar. \\
\hline 2012 & V Congreso Mundial por los Derechos de la Infancia y la Adolescencia. \\
\hline 2012 & Reunión Internacional de Primera Infancia. \\
\hline 2015 & Conferencia Global sobre Educación en la Primera Infancia. \\
\hline 2015 & Cumbre de las Naciones Unidas, Objetivos del Desarrollo Sostenible. \\
\hline 2016 & $\begin{array}{l}\text { Encuentro Internacional de Educación Infantil “El cuerpo en juego en la educación de la } \\
\text { primera infancia”. }\end{array}$ \\
\hline 2017 & $\begin{array}{l}\text { Asamblea Mundial y Conferencia Internacional “Las primeras relaciones de la infancia: la } \\
\text { base para un futuro sostenible”. }\end{array}$ \\
\hline
\end{tabular}

Fuente: elaboración propia

\section{Atención y educación de la primera infancia}

El foco de atención de la calidad educativa en la primera infancia es realmente una apuesta y un compromiso internacional reciente. Se empezó a considerar prominente a partir de la celebración del Compromiso Hemisférico por la Educación de la Primera Infancia, que se realizó en Colombia, en 2007. Allí se acordó lo siguiente:

La educación de la primera infancia es fundamental en el proceso de desarrollo integral del niño o la niña desde el nacimiento hasta los ocho años, y reconocemos que se brinda de forma variable entre los países pero debe incluir el esfuerzo conjunto de la familia, la escuela y la comunidad para el desarrollo de políticas públicas efectivas, así como la contribución de los distintos sectores de la sociedad. (OEI, 2007, p. 1)

En consecuencia, se plantea el desarrollo de marcos de orden legal, jurídicos y dispositivos de financiamiento a escala latinoamericana, para asegurar la ejecución de políticas de primera infancia, siendo prioritario el aumento de cobertura en educación de calidad y la atención integral. Posteriormente, en la Conferencia Mundial sobre Atención y Educación de la Primera Infancia (AEPI), celebrada en 2010, se reafirmó en uno de los objetivos globales que la AEPI es un derecho de todos los niños, puesto que forma parte de la base del desarrollo. En este orden de ideas, la AEPI se define como: 
[...] las acciones orientadas al cuidado y la protección de los niños, es decir, aquellas acciones dirigidas a preservar la vida, favorecer el crecimiento sano y adecuado — cuidados sanitarios, vacunación, nutrición, higiene-y promover la estimulación e interacción social que propicie el aprendizaje. Desde esta concepción, las acciones de protección, cuidado y educación durante la primera infancia se presentan como elementos interrelacionados e interactivos para fomentar el desarrollo de los niños. (Unesco y oEI, 2010, p. 16)

Por consiguiente, la calidad educativa en la educación inicial requiere un abordaje educativo y pedagógico holístico, ya que convergen varias aristas, entre ellas la atención, el cuidado, la protección, el bienestar y el desarrollo integral de la primera infancia. La educación inicial no está focalizada en el asistencialismo para atender de manera exclusiva la salud, la nutrición, la vacunación y la higiene. Requiere una perspectiva y un enfoque complejo, dinámico y contextual, que dé respuesta a la variabilidad y a la identidad histórico-cultural propia de la infancia y, por supuesto, de la primera infancia.

La propia naturaleza polisémica de la calidad educativa y la construcción social que enmarca a la primera infancia permiten afirmar y reconocer la evidencia de infancias, por ser una etapa o ciclo de vida que necesita una connotación en plural, no homogeneizadora ni idéntica en la sociedad latinoamericana, y mucho menos en el ámbito mundial. De manera que al abordarse en el contexto educativo, no es posible universalizar ni uniformar las políticas, estrategias, proyectos y programas para atender su desarrollo. En este sentido, en Colombia, el Ministerio de Educación Nacional (MEN, 2014) manifiesta que

[...] la niñez se define y asume en cada sociedad de manera distinta según características específicas, dadas por esa naturaleza diversa que configura la existencia y la subjetividad respecto a ellas y ellos. Es así como no existe una única infancia. Las infancias son múltiples y diversas, dependiendo de los distintos espacios culturales en los que se encuentran las niñas y los niños y según los procesos de transformación de esta noción que se manifiestan en las prácticas de atención, cuidado y educación de las sociedades. (p. 13)

Por tal razón, la calidad educativa en la educación inicial es garantía del derecho a la educación y al desarrollo infantil de calidad. Aquí convergen varios atributos para potenciarlo, como son: el cuidado, la atención, la educación y la concepción de desarrollo. Este último es considerado por el MEN (2009b) como un "proceso de cambios no lineal, continuo, e integral producto de la multiplicidad de experiencias 
corporales, emocionales, sociales y cognitivas que obtienen los niños al interactuar el mundo, que los rodea" (pp. 14-15). Es relevante precisar que los cuatro atributos mencionados configuran la identidad y lógica de la educación inicial desde una perspectiva para la AEPI, manifestada por la Conferencia Mundial de Desarrollo Infantil Temprano (2010). En la tabla 6 se describe cada uno de los atributos de la AEPI, tomando como referencia el marco conceptual de la Conferencia Mundial sobre la AEPI (2010), Kisilevsky y Roca (2013) y Marco (2014).

Tabla 6. Atributos de la AEPI

\begin{tabular}{|c|c|}
\hline \multicolumn{2}{|r|}{ Atributos de la atención y educación de la primera infancia } \\
\hline Atributo & Definición \\
\hline Cuidado & $\begin{array}{l}\text { "Atención directa que implica una relación inter-personal e incluye acciones } \\
\text { tendientes a propiciar el crecimiento sano y adecuado integrando aspectos de } \\
\text { nutrición, sanitarios, subsistencia, bienestar, estimulación temprana, socio-emo- } \\
\text { cionales” (Marco, 2014, p. 16). }\end{array}$ \\
\hline Atención & $\begin{array}{l}\text { "Procesos de educación, higiene y nutricionales, que posibilitan el desarrollo del } \\
\text { niño en un entorno sano que propicie y potencialice el bienestar cognitivo y so- } \\
\text { cioemocional" (Marco, 2014, 20). }\end{array}$ \\
\hline Desarrollo & $\begin{array}{l}\text { "Proceso de reconstrucción y reorganización permanente [...]. El desarrollo cogniti- } \\
\text { vo, lingüístico, social y afectivo de los niños no es un proceso lineal. Por el contra- } \\
\text { rio, se caracteriza por un funcionamiento irregular de avances y retrocesos [...]. No } \\
\text { tiene un principio definitivo y claro, es decir, no inicia desde cero [...]. El desarrollo } \\
\text { no parece tener una etapa final, en otras palabras, nunca concluye, siempre podría } \\
\text { continuar" (MEN, 2009, pp. } 16 \text { y 18). Es producto de multiplicidad de experiencias } \\
\text { otorgadas por las variables sociales, culturales, educativas, económicas y políticas } \\
\text { en una sociedad determinada. }\end{array}$ \\
\hline $\begin{array}{l}\text { Educación } \\
\text { de la } \\
\text { primera } \\
\text { infancia }\end{array}$ & $\begin{array}{l}\text { “Considera el periodo de cuidado, atención y educación de niños menores de } 6 \text { años } \\
\text { que se produce fuera del ámbito familiar. Esto incluye modalidades de atención } \\
\text { destinadas a niños desde las primeras semanas de vida hasta su ingreso en la es- } \\
\text { cuela primaria” (oEI, 2013, p. 4). Es más amplio que la preescolarización, pues } \\
\text { aborda el aprendizaje que se da por medio de la estimulación temprana, la orien- } \\
\text { tación y una amplia gama de actividades y oportunidades para el desarrollo bio- } \\
\text { psico-social del niño. }\end{array}$ \\
\hline
\end{tabular}

Fuente: elaboración propia 
Paralelamente, los atributos de la AEPI deben estar en congruencia con la neurociencia, en especial la neuroeducación: una nueva línea de pensamiento y acción que tiene como principal objetivo acercar a los agentes educativos a los conocimientos relacionados con el cerebro y el aprendizaje (Campos, 2010, p. 10).

\section{Los principales desafíos de la calidad educativa en la educación inicial}

El panorama descrito evidencia, sin duda, que el marco internacional, y en especial el compromiso en Latinoamérica en torno a la educación inicial, ha trascendido a escala no solo educativa, sino también social, cultural, económica y política. Aquí el objetivo macro es el de garantizar el derecho a la educación de calidad para todos. Sin embargo, la educación inicial desde la perspectiva de la AEPI no es un tarea fácil, inmediata y de responsabilidad exclusiva de los organismos internacionales, ya que, como se ha argumentado, su proceso de conceptualización, caracterización, gestión y estrategias de acción, entre otros, ha llevado a múltiples reuniones, conferencias, simposios, talleres, foros, en los cuales la infancia y la primera infancia ha logrado un estatus y relevancia intransferible. Esto se refleja en los marcos de política en América Latina, lo que implica que la AEPI requiera un enfoque holístico que reconozca la responsabilidad colectiva y compartida por los organismos internacionales, gubernamentales, dirigentes educativos, docentes, padres familia, es decir, la comunidad educativa local, nacional e internacional.

Dicho enfoque implica repensar las experiencias significativas que se le brindan a los niños en su cotidianidad escolar, las formas y los canales de interacción, las mediaciones y los abordajes pedagógicos, los programas y proyectos para favorecer el cuidado y el desarrollo, el diseño de políticas a largo plazo, entre otros. A su vez, propicia un análisis en torno a otras temáticas complejas y de gran envergadura en la voluntad y los logros expuestos en las políticas educativas, como son: la relación entre atención y educación, el acceso a la educación, la igualdad de oportunidades, la calidad educativa y, en especial, el derecho a la educación para todos.

En este orden de ideas, el desarrollo y la claridad en las políticas educativas relacionadas con la calidad educativa en atención y desarrollo de la primera infancia no son suficientes, precisamente porque su cuidado y abordaje requieren una perspectiva dinámica y compleja en sí misma. Esto lleva a que de manera constante las directrices planteadas se analicen y retroalimenten, para enfrentar y resolver los desafíos que se generan. Entre estos se encuentran: 
1. Aumentar la cobertura y la universalización de la calidad educativa de forma significativa para los niños menores de tres años, como quedó enmarcado en el proyecto Metas Educativas 2021. Según Blanco (2014):

La universalización [...] implica que [...] los Estados aseguren plazas suficientes para la educación de los niños y niñas que lo requieran [...]. Aumentar el acceso de los menores de 3 años es un desafío mayor, dados los niveles actuales de cobertura, que exige una mayor responsabilidad del Estado en términos de políticas, inversión y recursos humanos. (p. 28)

2. Continuar interpretando las necesidades y dificultades evidenciadas en los marcos normativos existentes en la región latinoamericana, analizando los proyectos, los programas y las estrategias implementadas, las cuales han estado encaminadas a fortalecer la atención y el desarrollo de la primera infancia. De acuerdo con el marco conceptual de la Conferencia Mundial sobre la AEPI (2010):

Son muchos los países que no han adoptado las decisiones de política necesarias para dar atención y educación a los niños menores de tres años. Pocos países han creado marcos nacionales para la financiación, coordinación y supervisión de programas de AEPI para niños de 0 a 3 años de edad [...]. Se estima frecuentemente que la organización de la atención y la educación de los niños menores de tres años es una responsabilidad de las familias o del sector privado. (Unesco, 2010b, p. 6)

3. Repensar continuamente los escenarios, las estrategias y los materiales pedagógicos a los que convoca la educación inicial, que se dan en el presente y determinan el horizonte del niño. Para esto se requiere una perspectiva flexible que implemente un abordaje dinámico, complejo y de mayor calidad. La Conferencia Mundial, en su informe para América Latina y el Caribe para la AEPI (2010), expresa:

Se espera que los materiales pedagógicos acordes con los procesos educativos de la primera infancia se adecúen a la cultura de los niños y niñas, sean seguros, estimulen su curiosidad y capacidad de exploración, sean adecuados a los diversos momentos de desarrollo y propicien interacciones y experiencias de aprendizaje significativas. (Unesco, 2010b, p. 49)

4. Fortalecer la investigación referente a la calidad educativa y realizar diagnósticos acerca de la AEPI, que permitan implementar procesos de planeación, financiación, implementación y supervisión, acordes con las demandas de la región latinoamericana. Esto es crucial para enmarcar y revalidar de forma sólida el desarrollo y los 
análisis de las políticas educativas con garantías a mediano y largo plazo. En este sentido, la Conferencia Mundial, en su informe para América Latina y el Caribe para la AEPI (2010), manifiesta:

En los documentos normativos de los países — políticas, currículum, regulaciones- no suele haber una definición explícita de calidad, pero existen esfuerzos de distinta envergadura para definir indicadores o estándares de calidad que orienten la evaluación de programas y servicios que suelen ser de carácter sectorial [...]. La revisión de algunas propuestas de los países permite afirmar que los indicadores están más centrados en insumos y procesos, que en resultados e impactos en términos del desarrollo, bienestar, aprendizaje y participación de los niños, o en la participación y grado de satisfacción de las familias y comunidades, entre otros. (Unesco, 2010b, pp. 46-47)

5. Asumir la calidad educativa en la educación inicial desde una perspectiva holística. Este punto lleva al establecimiento de políticas para la AEPI acordes con los contextos socioculturales de cada región latinoamericana. Al respecto, la Unesco, basada en un estudio del Instituto Internacional de Planeamiento de la Educación, indica que "si bien la primera infancia se incluye en todos los planes de educación, la mayoría de estos no se ciñen a un enfoque holístico de la AEPI, que integre la atención, la salud, la educación y la nutrición” (Unesco, 2010, p. 6).

6. Analizar y modificar las políticas, los programas y los proyectos para la primera infancia, focalizados en acciones parcializadas que tomen como referente un modelo universal de atención y desarrollo, que a su vez reconozca, valore y resalte el derecho de una educación para todos y la diversidad en la sociedad.

La atención y educación en la primera infancia, desarrollada desde una perspectiva de derechos humanos, no solamente tiene la posibilidad de preparar a las personas para su vida futura y para construir mejores condiciones económicas, sino además para promover la paz dentro de la comunidad y las naciones, para promover la equidad, estimular la movilidad intergeneracional y superar la pobreza. (Muñoz, 2012, p. 8)

7. Eliminar la brecha entre diversos sectores (salud, jurídico, protección social) y la educación inicial, para que esta asuma su papel protagónico y decisivo, y se logre la articulación intersectorial respecto al cuidado, la atención y el desarrollo de la primera infancia. 
El liderazgo en torno a la gestación y los dos primeros años de vida de los niños lo tienen los sectores salud, trabajo, seguridad y protección social. Si bien las corrientes de cambio buscan [...] que otros sectores como el de educación, registro civil, vivienda, medio ambiente, cultura, mujer y familia participen en el diseño de lineamientos para el desarrollo de la infancia, esto es aún muy desigual en la región. (Palacio y Castañeda, 2009, p. 46)

8. Invertir y apostar a la cualificación de los docentes, desde su formación, como profesionales para la AEPI, de modo que modifique el quehacer que actualmente se evidencia en los docentes en ejercicio.

Los países iberoamericanos claman por más y mejor formación de sus agentes educativos y comunitarios encargados de la atención a la primera infancia. Una norma que parece instalada en algunos países es que, a menor edad de los niños, menor formación de sus educadores y agentes comunitarios. (Palacio y Castañeda, 2009, p. 47)

9. Vincular las prácticas del educador infantil desde la lógica de la neuroeducación, para así direccionar con sentido el desarrollo de los procesos sensoriales, representativos y racionales, y de esta forma se evidencie un nuevo estilo de atención, cuidado, enseñanza y aprendizaje para la infancia.

El proceso de desarrollo cerebral es gradual y por ello las propuestas de aprendizaje deben ir de lo más simple y concreto a lo más abstracto y complejo. En los niños más pequeños, las zonas subcorticales del sistema nervioso central ejercen una poderosa influencia en su forma de aprender, de comportarse, de comunicarse, de sentir las emociones vinculadas a los acontecimientos y de pensar. El movimiento, la impulsividad, la exploración, los cuestionamientos, la reactividad, el juego, la falta de control emocional, entre otras, son características esenciales de la primera infancia, que se van encauzando a medida que las zonas corticales, y principalmente la corteza prefrontal van limitando la acción de las zonas subcorticales. (Campos, 2010, p. 10)

Los desafíos anteriormente expuestos se articulan con la agenda 2030 construida sobre la base de los ods, con el fin de "reconducir al mundo por el camino de la sostenibilidad y la resiliencia”. Aquí la educación tiene un papel central para el cual los representantes de ciento sesenta y nueve Estados han planteado lo siguiente: "Nos comprometemos a proporcionar una educación de calidad, inclusiva e igualitaria a todos los niveles: enseñanza preescolar, primaria, secundaria y terciaria y formación 
técnica y profesional” (p. 31). Es de señalar que si bien las metas propuestas son a escala mundial, cada gobierno está en la libertad de plantear sus propias metas a partir de las realidades de sus países, construyendo las estrategias que requieran (lineamientos, políticas, leyes, etc.).

Todas las personas, sea cual sea su sexo, raza u origen étnico, incluidas las personas con discapacidad, los migrantes, los pueblos indígenas, los niños y los jóvenes, especialmente si se encuentran en situaciones de vulnerabilidad, deben tener acceso a posibilidades de aprendizaje permanente que las ayuden a adquirir los conocimientos y aptitudes necesarios para aprovechar las oportunidades que se les presenten y participar plenamente en la sociedad. (Fundación Santillana, Universidad de Alcalá y Unesco, 2017, p. 31)

Es evidente que esta agenda señala la importancia de la educación inclusiva y equitativa, que crea las posibilidades de aprender durante toda la vida. Este aspecto lleva a la necesidad de estar atentos a descubrir si los alumnos desarrollan las competencias pertinentes para asumir los retos que les presenta la sociedad.

\section{Conclusiones}

La educación inicial ha sido importante desde el siglo xx. Viene adoptando nombres como educación preprimaria, educación preescolar, educación parvularia, educación infantil y educación en la primera infancia. Esto muestra la trascendencia del concepto desde 1990, que fue fundamental para la Conferencia Mundial de Educación para todos, en Jomtien. Allí se reafirmó la importancia del periodo prenatal y los primeros años de vida para el desarrollo de las potencialidades del ser humano. Además, se resaltó el derecho a la educación como elemento positivo en el proceso de crecimiento de los niños, y se destacó la contribución que tiene en la sociedad, puesto que posibilita la equidad y contribuye en el desarrollo de los países.

Al señalar la necesidad de destacar la preponderancia de la calidad en la educación inicial desde la perspectiva de la AEPI para América Latina, se destaca que aún dicha relación debe reflexionarse, pues cuestionar el pensamiento latinoamericano acerca de la calidad, específicamente en la educación inicial, devela la complejidad de su interpretación, además del abordaje y la intervención de los conceptos infancia y primera infancia. 
Actualmente, el concepto de calidad educativa se asume desde una perspectiva sistémica, multidimensional, y su naturaleza es sociocultural. Esto le otorga una particularidad de diversidad que trasciende el abordaje, la intervención y el análisis desde el enfoque cuantitativo, como es la creación de instituciones educativas, los recursos pedagógicos, la ampliación de cobertura, el acceso al sistema, entre otros. De igual manera, desborda la postura cualitativa, los procesos de enseñanza-aprendizaje, el abordaje pedagógico y las metodologías de evaluación y autoevaluación. En este orden de ideas, la calidad en la educación inicial lleva a un abordaje complejo para dar respuesta a la AEPI en el escenario latinoamericano, en el cual confluyen posturas ideológicas, pedagógicas, socioculturales, entre otras.

Así mismo, la evolución y el abordaje de la infancia han estado unidos al tiempo y al momento histórico-social de Latinoamérica y el mundo. Esto refleja heterogeneidad en su significado. Por consiguiente, el concepto de infancia es una construcción social en la que confluyen variables económicas, sociales, culturales, políticas y educativas.

La calidad en la educación inicial desde la perspectiva de la AEPI convoca a un proceso de coconstrucción entre el derecho a la educación y su garantía, por medio de las políticas educativas. Con esto se percibe un abordaje cíclico e inagotable, ya que al configurar y promover las políticas, reformas, programas y proyectos orientados a la población infantil, se debe tener en cuenta que son vectores fundamentales en el desarrollo de la sociedad y, por tanto, deben responder a las necesidades, las dificultades y los desafíos que conlleva la educación inicial. Indudablemente, dicha perspectiva necesita una conciencia colectiva que asuma la calidad en la educación inicial como política de Estado enmarcada desde las directrices internacionales, pero pensada para el contexto de cada país, de tal suerte que cada sujeto pueda mejorar su calidad de vida y sea una educación realmente para todos.

Las reformas y políticas educativas en América Latina han introducido cambios significativos en la conceptualización y el abordaje de la infancia, la primera infancia y la educación inicial. Sus mayores impactos se focalizan en procesos de acceso, cobertura e igualdad de oportunidades. Sin embargo, el mayor desafío latente en la región está asociado a la brecha existente entre el marco normativo a escala internacional y sus realidades educativas cotidianas, para garantizar la calidad de la educación inicial, en la cual se debe velar por el cuidado, la atención y el desarrollo, invitando a asumirlo desde una visión sistémica que garantice el desarrollo infantil integral en contexto.

Finalmente, apostarle a la calidad en la educación inicial es sinónimo de desarrollo y, ante todo, una garantía de derecho. Por lo tanto, debe continuar siendo un 
aspecto prioritario en las agendas de los organismos internacionales para superar las dificultades locales y los problemas comunes de las regiones latinoamericanas.

\section{Sobre las autoras}

Ángela María Jiménez Rojas. Psicóloga de la Universidad de Manizales, con Especialización en Pedagogía de la Universidad del Quindío y Maestría en Educación y Desarrollo Humano del Centro Internacional de Educación y Desarrollo Humano, en convenio con la Universidad de Manizales. Actualmente es doctorante en Ciencias de la Educación, Red de Universidades Estatales, CADE, de la Universidad del Quindío, institución en la cual es docente de carrera del programa de Licenciatura en Pedagogía Infantil. Ha publicado los siguientes libros: Aproximaciones conceptuales al estudio del desarrollo de las infancias. Una mirada desde diversas corrientes psicológicas (2018); El deportista lesionado: aproximación psicológica y aplicaciones prácticas para el educador físico y el técnico (2017); El pensamiento integral. Una perspectiva para enseñar en el aula (2016); Infancia y contexto sociocultural, desde una perspectiva vigotskiana (2015) e Infancia y cognición, un asunto de la epistemología genética (2015).

Luz Stela Quintana Hernández. Psicóloga con Especialización en Psicología de la Familia, de la Pontificia Universidad Javeriana, Cali, y magíster en Alta Dirección de Servicios Educativos de la Universidad de San Buenaventura, Cali. Actualmente es docente catedrática en la Universidad del Quindío, y docente de medio tiempo de la Universidad de San Buenaventura, Medellín, extensión Armenia. Sus temas de interés son: educación, primera infancia, políticas públicas y familia. Ha publicado los siguientes artículos: "Política pública de primera infancia: reconociendo el desarrollo y los talentos del ser humano" (2015) y "Las relaciones y el manejo del poder en el Ethos educativo" (2011). Su compromiso, participación y aportes en procesos de formación de las licenciaturas en Educación Especial y en Educación Infantil han sido reconocidos en el desarrollo del convenio marco de cooperación académica suscrito entre la Universidad Pedagógica Nacional y la Normal Superior Santiago de Cali.

\section{Referencias}

Aguerrondo, I. (2009). La calidad de la educación: ejes para su definición y evaluación. Madrid: Organización de Estados Iberoamericanos.

Alzate, M. P. (2003). La infancia: concepciones y perspectivas. Pereira: Editorial Papiro.

Bedoya, C. (2010). Amartya Sen y el desarrollo humano. Revista Nacional de Investigación. Memorias, 8(13), 277-288.

Bellamy, C. (2004). Estado mundial de la infancia 2005: la infancia amenazada. Nueva York: Unicef. Recuperado de https://www.unicef.org/spanish/publications/files/ SOWC_2005_(Spanish).pdf

Blanco, R. (2014). Inclusión educativa en América Latina: caminos recorridos y por recorrer. En A. Marchesi, R. Blanco y L. Hernández (Coords.), Avances y desafios de la educación 
inclusiva en Iberoamérica (pp. 11-35). Madrid: Organización de Estados Iberoamericanos para la Educación, la Ciencia y la Cultura.

Bodero, H. (2014). El impacto de la calidad educativa. Apuntes de Ciencias Sociales, 4(1), 112-117.

Cariola, L. (2013). La evaluación de aprendizajes, herramienta para mejorar la calidad educativa. En M. Kisilevsky y E. Roca (Coords.), Indicadores, metas y políticas educativas (pp. 61-79). Madrid: Organización de Estados Iberoamericanos para la Educación, la Ciencia y la Cultura.

Campos, A. (2010). Neuroeducación: uniendo las neurociencias y la educación en la búsqueda del desarrollo humano. Revista La Educ@ción, (143), 1-14. Recuperado de http://www.educoea.org/portal/La_Educacion_Digital/laeducacion_143/articles/neuroeducacion.pdf

Cejudo, R. (2006). Desarrollo humano y capacidades: aplicaciones de la teoría de las capacidades de Amartya Sen a la educación. Revista Española de Pedagogía, 64(234), 365-380.

Centro de Información de las Naciones Unidas. (s. f.). Los ocho objetivos del milenio. Recuperado de http://www.cinu.mx/minisitio/ODM8/los_8_objetivos_del_milenio/

Colombia. (8 de noviembre de 2006). Ley 1098 de 2006. Por la cual se expide el Código de la Infancia y la Adolescencia. Diario Oficial n..$^{46.446 . ~ B o g o t a ́ . ~}$

Consejo Nacional de Política Económica Social 109. (2007). Política Pública Nacional de Primera Infancia. Colombia por la Primera Infancia. Bogotá: Ministerio de la Protección SocialMinisterio de Educación Nacional-Instituto Colombiano de Bienestar Familiar.

Corrales, J. (1999). Aspectos políticos en la implementación de las reformas educativas. Santiago de Chile: Preal.

Chavarro, D., Vélez, M. I., Tovar, G., Montenegro, I., Hernández, A. y Olaya, A. (2017). Los Objetivos de Desarrollo Sostenible en Colombia y el aporte de la ciencia, la tecnología y la innovación. Recuperado de https://minciencias.gov.co/sites/default/files/objetivos_de_ desarrollo_sostenible_en_colombia_y_el_aporte_de_la_ctei_2.pdf

Delors, J. (1996). La educación encierra un tesoro: informe a la Unesco de la Comisión Internacional sobre la Educación para el Siglo XXI. Madrid: Santillana-Unesco.

Fundación Santillana, Universidad de Alcalá y Unesco. (2017). La nueva agenda educativa para América Latina: los objetivos para 2030. Recuperado de http://fundacionsantillana.com/ PDFs/860697.PDF

Gajardo, M. (1999). Reformas educativas en América Latina. Balance de una década. Santiago de Chile: Preal.

García, B. G. y Gallego, B. T. (2011). Una concepción abierta e interdisciplinar de la infancia. Revista Latinoamericana de Ciencias Sociales, Niñez y Juventud, 9(2), 17-25.

Guzmán, C. (2005). Reformas educativas en América Latina: un análisis crítico. Revista Iberoamericana de Educación, 36(8). Recuperado de https://rieoei.org/historico/ deloslectores/882Guzman.PDF

Jaramillo, L. (2007). Concepción de infancia. Zona Próxima, (8), 108-123. 
Kisilevsky, M. y Roca, E. (Coords.) (2013). Indicadores, metas y políticas educativas. Madrid: Organización de Estados Iberoamericanos para la Educación, la Ciencia y la Cultura.

Marchesi, A. (2014). Retos y dilemas de la inclusión educativa. En A. Marchesi, R. Blanco y L. Hernández (Coords.), Avances y desafíos de la educación inclusiva en Iberoamérica (pp. 3745). Madrid: Organización de Estados Iberoamericanos para la Educación, la Ciencia y la Cultura.

Marchesi, A., Tedesco, J. C. y Coll, C. (2009). Calidad, equidad y reformas en la enseñanza. Madrid: Organización de Estados Iberoamericanos para la Educación, la Ciencia y la Cultura-Fundación Santillana.

Marchesi, A., Blanco, R. y Hernández, L. (2014). Avances y desafíos de la educación inclusiva en Iberoamérica. Madrid: Fundación Mapfre-Organización de Estados Iberoamericanos para la Educación, la Ciencia y la Cultura.

Marco, F. (2014). Calidad del cuidado y la educación para la primera infancia en América Latina. Igualdad para hoy y mañana. Madrid: Cepal-Programa para la Cohesión Social en América Latina. Recuperado de http://repositorio.cepal.org/bitstream/handle/11362/36822/ S1420230_es.pdf;jsessionid=6B285D30F3E4B61972C2269FA79DAB06? sequence $=1$

Ministerio de Educación Nacional de Colombia. (2009). Desarrollo infantily competencias en la primera infancia. Bogotá.

Ministerio de Educación Nacional de Colombia. (2009b) Guía operativa para la prestación del servicio de atención integral a la primera infancia. Bogotá.

Ministerio de Educación Nacional de Colombia. (2010). El reto es consolidar el sistema de calidad educativa. Al Tablero, (56). Recuperado de https://www.mineducacion.gov. co/1621/article-242097.html

Ministerio de Educación Nacional. (2014). Sentido de la educación inicial. Bogotá.

Muñoz, V. (2012). Derechos desde el principio. Atención y educación en la primera infancia. Global Campalgn for Education. Recuperado de https://web.oas.org/childhood/ES/Lists/Recursos\%20\%20Bibliografia/Attachments/13/1.pdf

Murillo, J y Cuenca, R. (2007). Construyendo consensos en torno al concepto de educación de calidad. Revista Electrónica Iberoamericana sobre Calidad, Eficacia y Cambio en Educación, 5(3). Recuperado de https://www.redalyc.org/pdf/551/55130501.pdf

Organización de las Naciones Unidas y Cepal. (2018). La Agenda 2030 y los Objetivos de Desarrollo Sostenible: una oportunidad para America Latina y el Caribe. Santiago de Chile. Recuperado de https://repositorio.cepal.org/bitstream/handle/11362/40155/24/ S1801141_es.pdf

Organización de Estados Iberoamericanos para la Educación, la Ciencia y la Cultura (2007). V Reunión de Ministros de Educación. Compromiso Hemisférico por la Educación de la Primera Infancia, Cartagena de Indias, Colombia. 
Organización de Estados Iberoamericanos para la Educación, la Ciencia y la Cultura. (2013). Proyecto iberoamericano para colaborar en la atención integral a la primera infancia y a la educación inicial. 2014-2017. Panamá: Ministerio de Educación de Panamá.

Organización de las Naciones Unidas. (1948). Declaración Universal de los Derechos Humanos. Recuperado de http://www.derechoshumanos.net/normativa/normas/1948-DeclaracionUniversal.htm

Organización de las Naciones Unidas. (2000). Declaración del Milenio. Nueva York: Asamblea General.

Palacios, J. y Castañeda, E. (2009). La primera infancia (0-6 años) y su futuro. Madrid: Organización de Estados Iberoamericanos para la Educación, la Ciencia y la Cultura.

Programa de las Naciones Unidas para el Desarrollo. (2010). Desarrollo de capacidades: texto básico del PNUD. Nueva York: Programa de las Naciones Unidas para el Desarrollo.

Programa de las Naciones Unidas para el Desarrollo. (s. f.). Objetivos de Desarrollo Sostenible. Recuperado de https://www.undp.org/content/undp/es/home/sustainable-development-goals.html

Real Academia Española. (2019). Calidad. Diccionario de la lengua española. Recuperado de https://dle.rae.es/calidad?m=form

Reimers, F. (2000). Educación, desigualdad y opciones de política en América Latina en el siglo XxI. Revista Latinoamericana de Estudios Educativos, 30(2), 11-42.

Romero, T. (2009). Políticas de primera infancia en Iberoamérica: avances y desafíos en el siglo Xxi. En J. Palacios y E. Castañeda (Coords.), La primera infancia ( $0-6$ años) y su futuro. Madrid: Organización de Estados Iberoamericanos para la Educación, la Ciencia y la Cultura.

Seibold, J. (2000). La calidad integral en educación, reflexiones sobre un nuevo concepto de calidad educativa que integre valores y equidad educativa. Revista Iberoamericana de Educación, (23). Recuperado de http://rieoei.org/rie23a07.htm

Schmelkes, S. (2009). Equidad, diversidad, interculturalidad: las rupturas necesarias. En A. Marchesi, J. C. Tedesco y C. Coll (Coords.), Calidad, equidad y reformas en la enseñanza (pp. 47-56). Madrid: Organización de Estados Iberoamericanos para la Educación, la Ciencia y la Cultura.

Tiana, A. (2009). Calidad, evaluación y estándares: algunas lecciones de las reformas recientes. En A. Marchesi, J. C. Tedesco y C. Coll (Coords.), Calidad, equidad y reformas en la enseñanza (pp. 113-123). Madrid: Organización de Estados Iberoamericanos para la Educación, la Ciencia y la Cultura.

Unesco. (1999). Proyecto principal de educación para América Latina y el Caribe. Santiago de Chile. Recuperado en: http://unesdoc.unesco.org/images/0011/001131/113160s.pdf

Unesco. (2010a). Atención y educación de la primera infancia, informe regional: América Latina y el Caribe. Santiago de Chile. Recuperado de http://unesdoc.unesco.org/ images/0018/001892/189212S.pdf 
Unesco. (2010b). Conferencia Mundial sobre Atención y Educación de la Primera Infancia: construir la riqueza de las naciones. Documento conceptual de la conferencia. Rusia. Recuperado de http://unesdoc.unesco.org/images/0018/001873/187376s.pdf

Unesco. (2014). América Latina y el Caribe. Revisión regional 2015 en la Educación para todos. Santiago de Chile. Recuperado de https://unesdoc.unesco.org/ark:/48223/ pfo000232701_spa

Unesco y Organización de Estados Iberoamericanos para la Educación, la Ciencia y la Cultura. (2010). Sistema de Información sobre los derechos del niño en la primera infancia en los países de América Latina. Marco teórico y metodológico. Recuperado de http://www.siteal.iipeoei.org/sites/default/files/siteal_libros_digitales_01_0.pdf

Unicef. (s. f.). Primera infancia. Recuperado de http://unicef.cl/web/primera-infancia/

Unicef. (1989). Convención sobre los derechos del niño. Madrid. Recuperado de https://www.ohchr.org/sp/professionalinterest/pages/crc.aspx

Unicef. (2005). La infancia amenazada. Estado mundial de la infancia. Definición de infancia. Recuperado de https://www.unicef.org/spanish/sowc05/childhooddefined.html

Unicef. (2006). Convención sobre los derechos del niño. Madrid. Recuperado de https://www. unicef.es/sites/unicef.es/files/CDN_06.pdf 